\title{
Emergence of a
} daptomycin-non-susceptible Enterococcus faecium strain that encodes mutations in DNA repair genes after high-dose daptomycin therapy

\author{
Takashi Matono ${ }^{1}$, Kayoko Hayakawa ${ }^{1 *}$, Risen Hirai ${ }^{2}$, Akira Tanimura ${ }^{2}$, Kei Yamamoto ${ }^{1}$, Yoshihiro Fujiya ${ }^{1}$, \\ Momoko Mawatari', Satoshi Kutsuna', Nozomi Takeshita', Kazuhisa Mezaki ${ }^{3}$, Norio Ohmagari ${ }^{1}$ \\ and Tohru Miyoshi-Akiyama ${ }^{4}$
}

\begin{abstract}
Background: An increasing number of reports have documented the emergence of daptomycin-nonsusceptible Enterococcus in patients during daptomycin therapy. Even though several mechanisms for daptomycin-nonsusceptibility have been suggested, the potential genetic mutations which might contribute to the daptomycin-nonsusceptibility are not fully understood.

Case presentation: We isolated a vancomycin-susceptible, daptomycin nonsusceptible Enterococcus faecium strain from a patient with acute lymphocytic leukemia who received high-dose daptomycin therapy for $E$. faecium endocarditis. Whole-genome sequencing analysis revealed mutations within genes encoding DNA repair proteins MutL and RecJ of the daptomycin-nonsusceptible Enterococcus strain which might have facilitated its emergence.

Conclusions: We identified the mutations of DNA mismatch repair genes in a clinical isolate of daptomycin nonsusceptible E. faecium which emerged in spite of high-dose daptomycin therapy. The finding implicates the possible association of DNA repair mechanism and daptomycin resistance. Careful monitoring is necessary to avoid the emergence of daptomycin non-susceptible isolates of E. faecium and particularly in cases of long-term daptomycin use or in immunocompromised patients.
\end{abstract}

Keywords: Daptomycin, E. faecium, Whole-genome sequence

\section{Background}

Daptomycin (DAP) is a lipopeptide antibiotic that exhibits potent activity against gram-positive bacteria, including vancomycin-resistant enterococci (VRE); however, an increasing number of reports have documented the emergence of daptomycin-nonsusceptible Enterococcus (DNSE) in patients during DAP therapy [1-4]. Even

\footnotetext{
*Correspondence: kayokohayakawa@gmail.com

1 Disease Control and Prevention Center, National Center for Global

Health and Medicine, 1-21-1 Toyama, Shinjuku-ku, Tokyo 162-8655, Japan

Full list of author information is available at the end of the article
}

though several mechanisms for daptomycin-non-susceptibility have been suggested $[5,6]$, the potential genetic mutations which might contribute to the daptomycinnonsusceptibility are not fully understood. In this report, we describe vancomycin-susceptible, daptomycin nonsusceptible Enterococcus (DNSE) faecium strain from a patient with acute lymphocytic leukemia who received high-dose DAP therapy. The whole-genome sequencing analysis revealed mutations within genes encoding DNA repair proteins MutL and RecJ. 


\section{Case presentation}

A 32-year-old Japanese man with Philadelphia chromosome-positive acute lymphocytic leukemia (ALL) developed fever during chemotherapy with dasatinib and doxorubicin with dexamethasone for treatment of ALL relapse approximately 3 months after hematopoietic stem cell transplantation. The patient's blood culture was positive for E. faecium, and, as he was allergic to vancomycin, teicoplanin therapy was initiated. Dasatinib and doxorubicin were discontinued immediately. The minimum inhibitory concentrations (MICs) of various antibiotics are listed in Table 1 (EFM01). Although the MIC of teicoplanin for the E. faecium strain was $\leq 2 \mathrm{mcg} / \mathrm{ml}$, and the patient's serum teicoplanin trough was maintained between 20 and $22 \mathrm{mcg} / \mathrm{ml}$, E. faecium was consistently detected in his blood cultures for more than 3 weeks. In addition, the patient was neutropenic during this period, with neutrophil counts between 300 and 830 neutrophils/ $\mathrm{ml}$.

After consulting the infectious disease service for recommendations on treating the persistent $E$. faecium infection, the treatment plan was modified to include gentamicin therapy $(1.3 \mathrm{mg} / \mathrm{kg}$ every $12 \mathrm{~h})$, and imaging studies and an endoscopy were ordered to identify the nidus of the persistent E. faecium bacteremia. A transthoracic echocardiogram subsequently revealed a vegetation, measuring a few millimeters in size, on the patient's aortic valve. Meanwhile, chest and abdominal CT scans detected a thickened colon wall, but no other lesions, and a PET scan failed to identify a potential source of the infection. A colonoscopy, however, revealed erosion throughout the colonic mucosa, which was considered consistent with graft versus host disease and was considered the likely entry site of E. faecium into the bloodstream.

As the E. faecium bacteremia persisted for 2 weeks after initiation of the gentamicin therapy (gentamicin MIC was $16 \mathrm{mcg} / \mathrm{ml}$ ) in combination with teicoplanin, the patient was switched to $10 \mathrm{mg} \mathrm{kg}^{-1} \mathrm{day}^{-1}$ DAP [DAP; Etests indicated that the MIC of DAP for the $E$. faecium strain was $4 \mathrm{mcg} / \mathrm{ml}$ as in Table 1 (EFM01)].
After initiation of DAP therapy, the patient's fever subsided and subsequent blood cultures were negative. As a result, after receiving the initial dose of DAP for 18 days, the dose was reduced to $6 \mathrm{mg} \mathrm{kg}^{-1}$ day $^{-1}$. However, 1 day after reducing the dose, the patient developed fever again and his blood culture tested positive for E. faecium (DAP MIC, per Etest: $256 \mathrm{mcg} / \mathrm{ml}$ ). The MICs for other antibiotics are listed in Table 1 (EFM02). DAP was therefore discontinued, and treatment with intravenous linezolid (600 mg every $12 \mathrm{~h}$ ) was initiated. While blood cultures were negative after 2 days of linezolid therapy, the patient unfortunately passed away owing to exacerbation of the ALL at 26 days after initiation of treatment with linezolid.

Molecular analysis of the daptomycin-susceptible (EFM01; isolated prior to the initiation of DAP) and daptomycin non-susceptible E. faecium (DNSE; EFM02) isolates was conducted in the Pathogenic Microbe Laboratory at the Research Institute of the National Center for Global Health and Medicine in Tokyo, Japan. The strains were cultured in brain heart infusion (BHI) broth overnight, and genomic DNA was purified using a DNeasy Blood \& Tissue kit (Qiagen, Venlo, Netherlands). The genomes of the two isolates were then subjected to MiSeq sequencing using Nextera XT library kits (Illumina, Inc., San Diego, CA, USA), according to the manufacturer's instructions. Approximately 1 million pair-end reads (301 base pairs [bp] $\times 2$ ) were obtained from each genome and analyzed using CLC Genomics Workbench software (CLC bio, Aarhus, Denmark). The reads from each isolate were trimmed by screening for base quality (quality score limit $=0.05$; reads that contained greater than two ambiguous nucleotides or that were less than $15 \mathrm{bp}$ in length were removed), and then used to generate de novo genome assemblies, respectively. Meanwhile, the contigs were used as the reference genome. The reads from each isolate were then mapped to the reference genome, and variants were detected using CLC Genomics Workbench program that is based on the algorithm developed by Smith and Waterman (1981) [7]. For these analyses, the following detection parameters were used: $95 \%$ coverage and more than 10 overlapping reads.

Table 1 Susceptibility profile of resistance genes of Enterococcus faecium isolates

\begin{tabular}{|c|c|c|c|c|c|c|c|c|c|c|c|c|c|c|c|}
\hline \multirow[t]{2}{*}{ Isolate } & \multicolumn{6}{|c|}{ Resistance genes } & \multicolumn{9}{|c|}{ Minimum inhibitory concentrations (MIC) $(\mu \mathrm{g} / \mathrm{ml}) /$ Interpretive criteria ${ }^{a}$} \\
\hline & $\operatorname{Aac}\left(6^{\prime}\right)-l i$ & Ant(6)-Ia & $\operatorname{Aph}\left(3^{\prime}\right)-I I I$ & ErmB & MsrC & TetM & PCG & ABPC & EM & MINO & VCM & TEIC & LVFX & LZD & DAP \\
\hline EFM01 & + & + & + & + & + & + & $\geq 16 / R$ & $\geq 16 / R$ & $\geq 8 / R$ & $8 / 1$ & $1 / \mathrm{S}$ & $\leq 2 / S$ & $\geq 8 / R$ & $\leq 2 / S$ & $4 / 5$ \\
\hline EFM02 & + & + & + & + & + & + & $\geq 16 / R$ & $\geq 16 / R$ & $\geq 8 / R$ & $8 / 1$ & $1 / S$ & $\leq 2 / S$ & $\geq 8 / R$ & $\leq 2 / S$ & 256/- \\
\hline
\end{tabular}

PCG penicillin G, ABPC ampicillin, MINO minocycline, VCM vancomycin, TEIC teicoplanin, LVFX levofloxacin, LZD linezolid, DAP daptomycin, $S$ susceptible, I intermediate, $R$ resistant

${ }^{a}$ MIC interpretive criteria, per the clinical and laboratory standards institute (CLSI; M100-S24) [20] 
Because the settings used can yield false-positive variants, each putative variant was manually confirmed by examining the mapping results. The resulting sequencing data were registered with the DNA Data Bank of JAPAN (DDBJ, accession number DRA03513). Furthermore, to annotate variants that were unique to the strains examined in this study, the genome sequence of E. faecium Aus0085 was used as a Ref. [8].

The MICs of multiple antimicrobials for the two $E$. faecium isolates, as well as the antimicrobial resistance genes encoded by these organisms, as identified by analysis of contigs using the ResFinder program [4], are summarized in Table 1. Comparison of the EFM01 and EFM02 genomes at SNP level based on whole genome sequencing indicated that EFM02 was derived from EFM01. While EFM02 contained 40 variants that were not present in EFM01, each of the variants identified in EFM01 were present in EFM02. The variants that resulted in amino acid substitutions within the genome of EFM02 compared to that of EFM01 are summarized in Table 2.

Notably, by comparing the genomes of the two E. faecium isolates, we detected mutations that were present in the genes encoding the DNA repair proteins MutL (mutL) and RecJ (recJ) of the DNSE strain, but not the DAP-susceptible parental strain. We, therefore, investigated whether the disruption of these genes affected the frequency of mutations in the E. faecium genome. For these analyses, each strain was cultured overnight in $2 \mathrm{ml}$ of BHI broth. The following day, $2 \mu \mathrm{L}$ of the resulting cultures was used to inoculate $2 \mathrm{ml}$ of BHI broth, respectively. The cultures were again incubated overnight, diluted in fresh broth, and plated on BHI agar. Subsequently, 11 distinct colonies of each strain (EFM01 and EFM02) were harvested, and whole-genome sequencing of these isolates was conducted, as described above. The reads obtained from each isolate were mapped to the respective parental genome and analyzed for the presence of newly acquired variants. Because the settings used can yield false positive variants, any variants that were also present in the parental genome were excluded, and each putative variant was manually confirmed by examining the mapping results.

There was only one newly acquired variant identified after analysis of the genomes of the 11 daptomycinsusceptible E. faecium (EFM01) after the bacteria had undergone 9.2 generations. Conversely, analysis of the genomes of the 11 DNSE (EFM02) isolates detected 49 variants after the bacteria had undergone 9.4 generations. These findings indicate that the observed alterations to the mutL and recJ genes may have resulted in a significant increase in the frequency of mutations in the EFM02 genome.

\section{Discussion}

In this report, we characterized a strain of E. faecium with high level of DAP resistance $(\mathrm{MIC}=256 \mathrm{mcg} / \mathrm{ml}$ ) that was isolated from a patient with ALL following 20 days of exposure to high-dose DAP $\left(10 \mathrm{mg} \mathrm{kg}^{-1} \mathrm{day}^{-1}\right)$ for treatment of E. faecium endocarditis. Subsequent genomic analyses indicated that this DNSE strain contained mutations within the known DNA repair genes mutL and recJ, which may have contributed to the acquisition of DAP resistance. Although dasatinib was reported to have effect on DNA repair pathways in human cancer cell lines [9], the association of DNA repair gene mutations of bacterial isolates with dasatinib or doxorubicin has not been reported to the best of our knowledge. In this case, dasatinib and doxorubicin were discontinued at the time of the first episode of E. faecium bacteremia, and thus, the patient was not receiving these drugs during DNSE emergence.

In a previous study of 42 cases of DNSE infection, which included five cases due to vancomycin-susceptible DNSE, only two VRE strains (4.2\%) exhibited DAP MICs $\geq 128 \mathrm{mcg} / \mathrm{ml}$ [10]. Meanwhile, the most common underlying disease associated with DNSE infection was hematologic malignancy (35\%), which was also present in the current case [10]. Indeed, immunosuppression and prior exposure to cephalosporins and metronidazole are considered independent predictors of infections caused by DNSE [11]. While in vitro analyses indicated that the acquisition of DAP resistance requires at least 6 days of exposure to DAP [6], the median duration of DAP exposure in previous case series of DNSE was 16-19 days [12, 13], which is similar to the duration of DAP treatment in the current case (20 days).

In recent reports of DNSE that developed during DAP therapy, patients received $6 \mathrm{mg} / \mathrm{kg}$ DAP $[1-3,14]$. Meanwhile, separate studies demonstrated that $\geq 8 \mathrm{mg} \mathrm{kg}^{-1} \mathrm{day}^{-1}$ of DAP resulted in improved clinical outcomes in cases of VRE blood stream infections, but that an even higher dose of DAP $\left(\geq 10 \mathrm{mg} \mathrm{kg}^{-1}\right.$ day $\left.^{-1}\right)$ might be required to prevent the development of DAP resistance $[6,15]$. In the current case, however, DNSE survived high-dose DAP therapy $\left(10 \mathrm{mg} \mathrm{kg}^{-1}\right.$ day $\left.^{-1}\right)$. While recent studies suggest that increases in DAP MICs are associated with decreases in the MIC of beta-lactams [16], further investigation is required to assess whether the inclusion of beta-lactams such as ampicillin might help prevent the development of DNSE. Furthermore, a recent meta-analysis indicated that linezolid is more effective than is DAP for treatment of VRE bacteremia and that linezolid was associated with decreased rates of mortality [17]. However, the side effects associated with this antimicrobial, particularly adverse hematologic reactions, hinder its long-term use. 


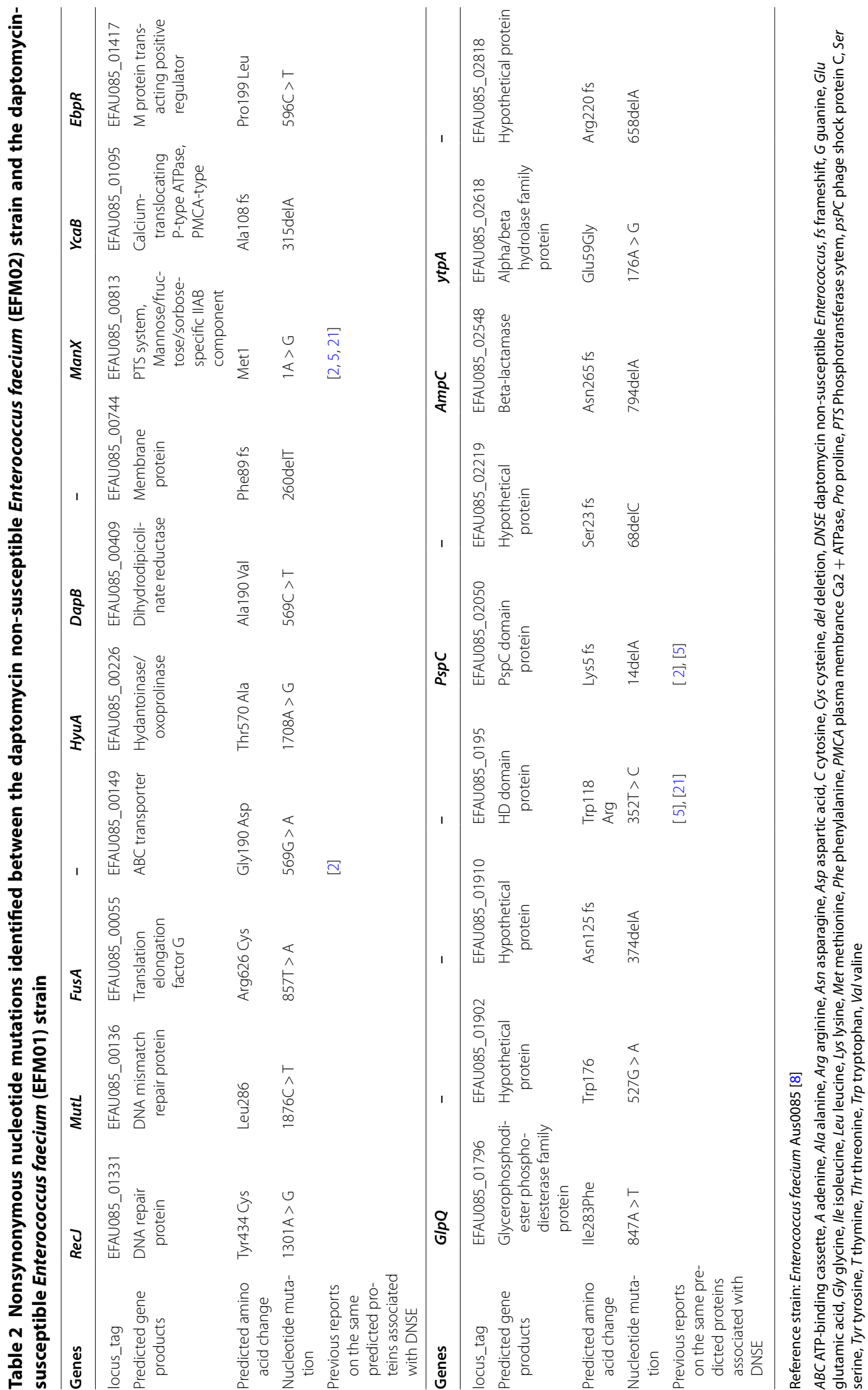


The mechanisms underlying DAP non-susceptibility in enterococci are not fully understood, but recent reports suggest the involvement of the cardiolipin synthase enzyme as well as several genetic pathways, including those associated with cell membrane phospholipid metabolism and the response of the bacterial cell-envelope to antibiotics [5]. We did not identify mutations that have been previously determined to confer daptomycin non-susceptibility in E. faecium, such as liaFSR, yycFGHIJ, cardiolipin synthetase, or ezrA [5]. However, we identified multiple amino acid changes predicting gene products which were reported previously in daptomycin non-susceptibile E. faecium isolates by whole-genome analyses, as shown in Table 2 . In addition, we identified mutations within the DNA repair genes $m u t L$ and recJ that were unique to the DNSE strain and demonstrated that these mutations might have facilitated the emergence of spontaneous mutations during subculturing. We propose that this increased frequency of mutation might have led to the observed emergence of the DNSE phenotype. Contrary to this hypothesis, in a previous study, Willems et al. failed to detect demonstrable hypermutator phenotypes in oxazolidinone-resistant or -susceptible E. faecium isolates with mutations in the mutLS locus [18]. However, it is possible that the distinct phenotypes associated with alterations in the mutL gene could be due to differences in the genetic position of the individual mutL mutations [18]. Meanwhile, the recJ gene encodes a $5^{\prime}-3^{\prime}$ single-stranded DNA-specific exonuclease that was reported to be associated with illegitimate recombination [19]. To the best of our knowledge, there have been no reports that have examined mutations in the recJ gene or the role of this protein in Enterococcus spp. Further studies are therefore are needed to reveal the association, if any, between mutations in recJ and the development of DNSE.

\section{Conclusions}

In conclusion, we isolated a strain of vancomycin-susceptible, DAP non-susceptible E. faecium, which survived exposure to high-dose $\left(10 \mathrm{mg} \mathrm{kg}^{-1}\right.$ day $\left.^{-1}\right)$ DAP for treatment of $E$. faecium endocarditis. Whole-genome sequencing revealed mutations within the mutL and recJ genes of the DNSE strain, while in vitro analyses demonstrated that the DNSE strain exhibited higher rates of spontaneous mutation than did the parental strain. Our findings demonstrate that careful monitoring is necessary to avoid the emergence of DAP non-susceptible isolates of E. faecium, in spite of high-dose therapy, and particularly in cases of long-term DAP use or in immunocompromised patients such as those with hematological malignancy. Our study did not include the demonstration of the relationship of these DNA repair genes mutations with phenotypic changes, and we were unable to determine the exact mechanism of resistance. Further investigation is necessary to elucidate the mechanism by which E. faecium acquires DAP resistance, as well as the contribution of mutations in the DNA mismatch repair genes $m u t L$ and recJ to this process.

\section{Abbreviations}

DAP: daptomycin; RE: vancomycin-resistant enterococci; DNSE: daptomycinnonsusceptible Enterococcus; ALL: acute lymphocytic leukemia; MIC: minimum inhibitory concentration; BHI: brain heart infusion.

\section{Authors' contributions}

TM and KH drafted the manuscript. KM carried out the microbiological analyses. TMA carried out the molecular genetic studies. RH, AT, KY, YF, MM, SK, NT and NO helped to draft the manuscript. All authors read and approved the final manuscript.

\section{Author details}

${ }^{1}$ Disease Control and Prevention Center, National Center for Global Health and Medicine, 1-21-1 Toyama, Shinjuku-ku, Tokyo 162-8655, Japan. ${ }^{2}$ Department of Hematology, National Center for Global Health and Medicine, Tokyo, Japan. ${ }^{3}$ Microbiology Laboratory, National Center for Global Health and Medicine, Tokyo, Japan. ${ }^{4}$ Pathogenic Microbe Laboratory, Research Institute, National Center for Global Health and Medicine, Tokyo, Japan.

\section{Availability of data and materials}

The dataset supporting the conclusions of this is included with in the article.

\section{Acknowledgements and Funding}

This study was supported by Grants for International Health Research (26S-120)

\section{Competing interests}

The authors declare that they have no competing interests.

\section{Consent to publish}

Written informed consent was obtained from the patient for publication of this case report and any accompanying images.

Received: 1 December 2015 Accepted: 22 March 2016

Published online: 01 April 2016

\section{References}

1. Arias CA, Torres HA, Singh KV, Panesso D, Moore J, Wanger A, Murray BE. Failure of daptomycin monotherapy for endocarditis caused by an Enterococcus faecium strain with vancomycin-resistant and vancomycinsusceptible subpopulations and evidence of in vivo loss of the vanA gene cluster. Clin Infect Dis. 2007;45(10):1343-6.

2. Humphries RM, Kelesidis T, Tewhey R, Rose WE, Schork N, Nizet V, Sakoulas G. Genotypic and phenotypic evaluation of the evolution of high-level daptomycin nonsusceptibility in vancomycin-resistant Enterococcus faecium. Antimicrob Agents Chemother. 2012;56(11):6051-3.

3. Kelesidis T, Tewhey R, Humphries RM. Evolution of high-level daptomycin resistance in Enterococcus faecium during daptomycin therapy is associated with limited mutations in the bacterial genome. J Antimicrob Chemother. 2013;68(8):1926-8.

4. Zankari E, Hasman H, Cosentino S, Vestergaard M, Rasmussen S, Lund O, Aarestrup FM, Larsen MV. Identification of acquired antimicrobial resistance genes. J Antimicrob Chemother. 2012;67(11):2640-4.

5. Diaz L, Tran TT, Munita JM, Miller WR, Rincon S, Carvajal LP, Wollam A Reyes J, Panesso D, Rojas NL, et al. Whole-genome analyses of Enterococcus faecium isolates with diverse daptomycin MICs. Antimicrob Agents Chemother. 2014;58(8):4527-34. 
6. Werth BJ, Steed ME, Ireland CE, Tran TT, Nonejuie P, Murray BE, Rose WE, Sakoulas G, Pogliano J, Arias CA, et al. Defining daptomycin resistance prevention exposures in vancomycin-resistant Enterococcus faecium and E. faecalis. Antimicrob Agents Chemother. 2014;58(9):5253-61.

7. Smith TF, Waterman MS. Identification of common molecular subsequences. J Mol Biol. 1981;147(1):195-7.

8. Lam MM, Seemann T, Tobias NJ, Chen H, Haring V, Moore RJ, Ballard S, Grayson LM, Johnson PD, Howden BP, et al. Comparative analysis of the complete genome of an epidemic hospital sequence type 203 clone of vancomycin-resistant Enterococcus faecium. BMC Genom. 2013;14:595.

9. Peng S, Sen B, Mazumdar T, Byers LA, Diao L, Wang J, Tong P, Giri U, Heymach JV, Kadara HN, et al. Dasatinib induces DNA damage and activates DNA repair pathways leading to senescence in non-small cell lung cancer cell lines with kinase-inactivating BRAF mutations. Oncotarget. 2016;7(1):565-79.

10. Wang G, Kamalakaran S, Dhand A, Huang W, Ojaimi C, Zhuge J, Yee LL, Mayigowda P, Surendraiah PK, Dimitrova N, et al. Identification of a nove clone, ST736, among Enterococcus faecium clinical isolates and its association with daptomycin nonsusceptibility. Antimicrob Agents Chemother. 2014;58(8):4848-54.

11. Judge T, Pogue JM, Marchaim D, Ho K, Kamatam S, Parveen S, Tiwari N, Nanjireddy P, Bheemreddy S, Biedron C, et al. Epidemiology of vancomycin-resistant enterococci with reduced susceptibility to daptomycin. Infect Control Hosp Epidemiol. 2012;33(12):1250-4

12. Storm JC, Diekema DJ, Kroeger JS, Johnson SJ, Johannsson B. Daptomycin exposure precedes infection and/or colonization with daptomycin nonsusceptible enterococcus. Antimicrob Resist Infect Control. 2012;1(1):19.

13. Wudhikarn K, Gingrich RD, de Magalhaes Silverman M. Daptomycin nonsusceptible enterococci in hematologic malignancy and hematopoietic stem cell transplant patients: an emerging threat. Ann Hematol. 2013;92(1):129-31.
14. Kanafani ZA, Federspiel JJ, Fowler VG Jr. Infective endocarditis caused by daptomycin-resistant Enterococcus faecalis: a case report. Scand J Infect Dis. 2007;39(1):75-7.

15. Whang DW, Miller LG, Partain NM, McKinnell JA. Systematic review and meta-analysis of linezolid and daptomycin for treatment of vancomycinresistant enterococcal bloodstream infections. Antimicrob Agents Chemother. 2013;57(10):5013-8.

16. Sakoulas G, Nonejuie P, Nizet V, Pogliano J, Crum-Cianflone N, Haddad F. Treatment of high-level gentamicin-resistant Enterococcus faecalis endocarditis with daptomycin plus ceftaroline. Antimicrob Agents Chemother. 2013;57(8):4042-5.

17. Balli EP, Venetis CA, Miyakis S. Systematic review and meta-analysis of linezolid versus daptomycin for treatment of vancomycin-resistant enterococcal bacteremia. Antimicrob Agents Chemother. 2014;58(2):734-9.

18. Willems RJ, Top J, Smith DJ, Roper DI, North SE, Woodford N. Mutations in the DNA mismatch repair proteins MutS and MutL of oxazolidinoneresistant or -susceptible Enterococcus faecium. Antimicrob Agents Chemother. 2003;47(10):3061-6.

19. Shiraishi K, Hanada K, Iwakura Y, Ikeda H. Roles of RecJ, RecO, and RecR in RecET-mediated illegitimate recombination in Escherichia coli. J Bacteriol. 2002;184(17):4715-21

20. CLSI: The clinical and laboratory standards institute. performance standards for antimicrobial susceptibility testing; Twentieth Informational Supplement. Approved Standard M100-S24. Wayne: CLSI; 2014.

21. Tran TT, Panesso D, Gao H, Roh JH, Munita JM, Reyes J, Diaz L, Lobos EA, Shamoo Y, Mishra NN, et al. Whole-genome analysis of a daptomycin-susceptible Enterococcus faecium strain and its daptomycin-resistant variant arising during therapy. Antimicrob Agents Chemother. 2013;57(1):261-8.

\section{Submit your next manuscript to BioMed Central and we will help you at every step:}

- We accept pre-submission inquiries

- Our selector tool helps you to find the most relevant journal

- We provide round the clock customer support

- Convenient online submission

- Thorough peer review

- Inclusion in PubMed and all major indexing services

- Maximum visibility for your research

Submit your manuscript at www.biomedcentral.com/submit

() Biomed Central 